\title{
Evaluation of Assembly Gap from 3D Laser Measurements via FEA Simulation
}

\author{
Xueshu Liu $\mathbb{D}^{,}{ }^{1}$ Junhao Chang, ${ }^{2}$ Yuxing Yang, ${ }^{2}$ Yiqi Wang, ${ }^{2}$ Yongjie Bao, ${ }^{2}$ and Hang Gao ${ }^{2}$ \\ ${ }^{1}$ School of Automotive Engineering, Dalian University of Technology, Dalian 116024, China \\ ${ }^{2}$ School of Mechanical Engineering, Dalian University of Technology, Dalian 116024, China \\ Correspondence should be addressed to Xueshu Liu; liuxs@dlut.edu.cn
}

Received 15 May 2018; Revised 26 August 2018; Accepted 5 September 2018; Published 1 October 2018

Academic Editor: Santiago Hernández

Copyright (c) 2018 Xueshu Liu et al. This is an open access article distributed under the Creative Commons Attribution License, which permits unrestricted use, distribution, and reproduction in any medium, provided the original work is properly cited.

\begin{abstract}
Due to the compliance and geometrical defects of composite parts, gaps exist between assembly components after the preassembly. Assembly requirements impose to fill these gaps to eliminate any unexpected internal stresses. Although it is identified as a problematic and expensive nonadded value stage, a gap measurement is still needed. This paper develops a numerical process for gap prediction before the assembly step. After the assembly components are scanned in a specified configuration, finite element meshes are created using the scanned data and the shape variations of each component caused by constraints and forces in different configurations are evaluated by finite element analysis. Assembly gaps are finally assessed by assembling the simulated preassembly shapes of all components. The feasibility of the proposed method is proved by an experiment.
\end{abstract}

\section{Introduction}

Due to the excellent properties, carbon fiber reinforced plastic (CFRP) has been widely used in aircraft structures in recent years. However, because of the complicated manufacturing processes and anisotropic property of CFRP as well, it is almost impossible to manufacture a composite product without geometrical deviation including processinduced deformation and thickness variations at present, especially for large ones. As a result, gaps are formed at the faying surfaces during the assembly process and prestress would be consequently introduced by the assembly forces, which would result in the development of cracks of delamination. To avoid such problems, assembly gaps should be filled to eliminate the undesirable prestress. Unfortunately, in the aviation industry, the usage of shim is carefully restricted to avoid increasing the weight of structure and lowering the load capacity of aircraft as a result. On the other hand, a deficiency of shim will affect the mechanical performance of the structure as reported in [1-6]. Therefore, the accurate measurement of assembly gap plays an important role in the shimming process.
Nevertheless, the assessment of the assembly gap of CFRP structures is not trivial. First, the aircraft structures are commonly designed as a closed or semiclosed region such as the wing box. So not all the gaps can be reached during assembly when a direct measurement method is adopted. Moreover, for accurate shimming, the gaps should be represented in $3 \mathrm{D}$, which is a mission impossible for direction measurement. Second, even assembly gaps can be evaluated by numerical methods like virtual assembly after scanning all the components and rebuilding their actual geometries; high-accuracy assessment of assembly gap is still a challenge for aircraft structures. Because some components are flexible since their thicknesses are usually much less than their length and width like the aircraft plate, they tend to deform under their own weight, which results in the shape variations when their space orientation change. In addition, the measurement shape is normally different from that in assembly configuration because the assembly process generates deformations due to the application of forces and restrictions at fixation points and the requirement of prestress assembly as well. Therefore, the difference between the measurement shape and assembly shape has to be taken into consideration so as 
to accurately evaluate the assembly gaps and remove any possible prestress generated during the assembly process.

So far, many efforts on the shape prediction of flexible part in different configurations have been done. For example, Ascione and Polini gave a solution to the problem of nonrigid freeform inspection by a coordinate measuring machine [7]. But it is expensive and time-consuming to manufacture such equipment. So the recent trends in inspection methods for compliant components are referred to as the simulated displacement method [8]. For instance, Abenhaim et al. proposed the iterative displacement inspection algorithm for the inspection of flexible parts [9]. Gentilini and Shimada reported a method for the shape inspection of a flexible assembly part by virtually mounting it into the assembly [10]. First, the dense measured mesh is smoothed and reduced to become suitable for finite element analysis (FEA). Then, the specific displacement boundary conditions are defined and applied for the FE simulation of the assembly process. After that, quality inspection of the simulated postassembly shape can be done using visualization tools. Radvar-Esfahlan and Tahan presented a method for nonrigid part inspection which was based on simulation using FEA [11]. An approach based on only partial views of a part was reported in [12]. The authors focused on the regions where inspection is required and used the concept of characteristic points which implies more simplicity for calculations and for the surface acquisition process. Instead of FEA, a spring-mass model was defined and used to simulate deformations in [13]. To improve the reliability of the FE analysis results, Abenhaim et al. proposed a boundary displacement constrained optimization using FEA [14-16], and Karganroudi et al. introduced an algorithm to automatically filter out sample points that are close to defects [17]. Thiebaut et al. presented a method to obtain the form deviation of flexible components from part measurements independently of the assembly configuration of use [18].

The abovementioned contributions focus on the differences of a flexible part while in different configurations. One more step goes ahead, and the assembly gaps can be evaluated by a virtual method. Lacroix et al. represented a numerical process to predict an assembly gap before an assembly step from component measurements [19]. The main issue relates to the integration of measuring data into finite element meshes created by using a nominal model. Different from them, in this study, a new procedure for assembly gap prediction is proposed. Starting from the measurement data (usually point cloud), finite element meshes of the flexible part are created and the deformations caused by forces and restrictions in different configurations are evaluated by using FEA. After the assembly shapes of all components are obtained, virtual assembly technology is employed to assess the assembly gaps. Finally, the accuracy of this method is validated by an experiment.

\section{Principle of the Method}

Assembly component is always measured in a given configuration, using an over-constrained measuring set-up. So the measured shape $S^{\mathrm{m}}$ corresponds to the theoretical shape $S^{\mathrm{d}}$, added to the form deviation $E^{\mathrm{f}}$ and the deformation under the combined effect of the measuring set-up and the component's own weight $E^{\mathrm{m} c}$, which can be expressed by

$$
S^{\mathrm{m}}=S^{\mathrm{d}}+E^{\mathrm{f}}+E^{\mathrm{m} c} .
$$

Note that the term $\left(S^{\mathrm{d}}+E^{\mathrm{f}}\right)$ represents the free state of a component, which is the shape it should have in the state of absence of gravity and any constraints.

Similarly, the shape of a component in assembly configuration $S^{\text {a }}$ can be given by

$$
S^{\mathrm{a}}=S^{\mathrm{d}}+E^{\mathrm{f}}+E^{\mathrm{a} c},
$$

where $E^{\mathrm{ac}}$ indicates the deformation under the combined effect of the assembly set-up and the component's own weight.

Note that $S^{\mathrm{d}}$ and $E^{\mathrm{f}}$ was kept constant no matter in what kind of configuration the component involves. So the relationship between $S^{\mathrm{m}}$ and $S^{\mathrm{a}}$ is given by

$$
S^{\mathrm{a}}=S^{\mathrm{m}}-E^{\mathrm{m} c}+E^{\mathrm{a} c} .
$$

The deformations caused by forces and restrictions in different configurations, namely, $E^{\mathrm{m} c}$ and $E^{\mathrm{ac}}$, can be assessed by FEA. After all the shapes of components in assembly configuration are obtained, assemble them virtually and gaps can be clearly defined.

\section{Mesh Generation from Point Cloud}

After scanning a flexible part in a specified configuration with a laser scanner, a point cloud $\tilde{P}$ representing surfaces of the part is obtained. Postprocessing steps are usually needed because $\tilde{P}$ suffers from noise, which includes physical phenomena and hardware-related issues [10]. Such noise can be removed by using mean curvature flow, normal field diffusion algorithms, and so on. After noise removal, a clean point cloud $P=\left\{\left.p_{i} \in R^{3}\right|_{i=1,2, \ldots}\right\}$ is got and used for assembly shape prediction by FEA.

To create finite element meshes from $P$ for simulation, a procedure was proposed and shown in Figure 1. First, principal component analysis is performed on $P$ and the normal direction $\mathbf{n}$ of $P$ is computed. A plane $\Phi(\mathbf{n}, \widehat{p})$ is then defined by $\mathbf{n}$ and $\widehat{p} \in R^{3}$ (Figure $1(\mathrm{~b})$ ). A map $f: P \rightarrow \bar{P}$, which is given by (4), is used to project $P$ onto $\Phi$ and $\bar{P}=$ $\left\{\left.f\left(p_{i}\right)\right|_{\forall p_{i} \in P}\right\}$ is yielded.

$$
f: \mathbf{p}_{\mathbf{i}}+\frac{\mathbf{n} \cdot \vec{p}_{i} \widehat{p} \cdot \mathbf{n}}{\|\mathbf{n}\|^{2}},
$$

where $\mathbf{p}_{\mathbf{i}}$ indicates the Euclidean vector $\overrightarrow{o p}_{i}$ and $o$ represents the point $(0,0,0)$ in $R^{3}$. 


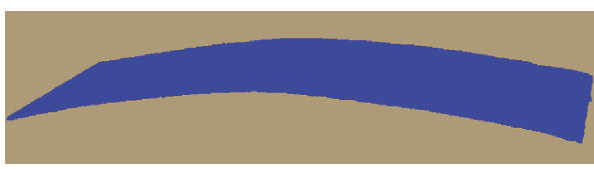

(a)

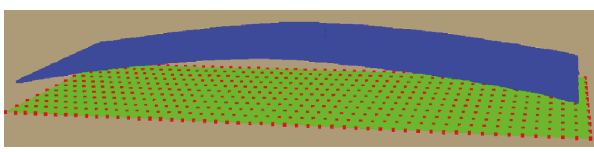

(c)

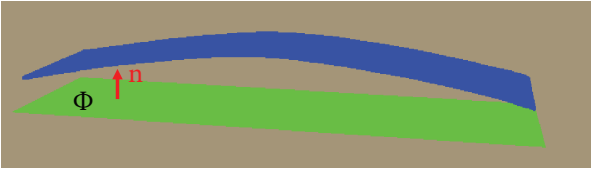

(b)

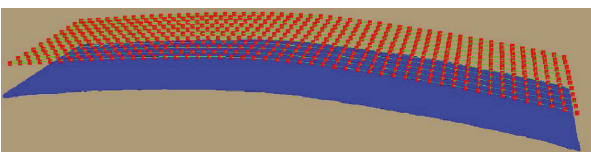

(d)

Figure 1: Procedure of mesh generation from point cloud. (a) Point cloud P. (b) Projection plane Ф. (c) Sampling points Q (red). (d) Mesh model.

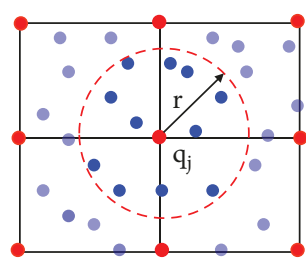

- $\left\{\mathrm{q}_{\mathrm{j}}\right\}$

- $\left\{\mathrm{f}\left(\mathrm{p}_{\mathrm{i}}\right)\right\}$

FIgURE 2: Illustration of average distance computation.

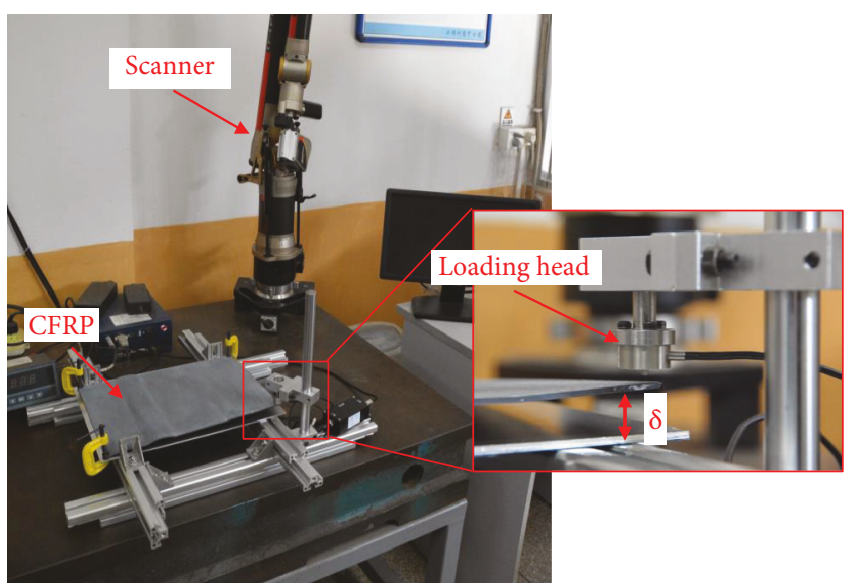

FIGURE 3: Experiment set-up.

The distance $D\left(p_{i}\right)$ from $p_{i} \in P$ to $\Phi$ is computed by

$$
D\left(p_{i}\right)=\frac{\mathbf{n} \cdot \vec{p}_{i} \hat{p}}{\|\mathbf{n}\|}
$$

Next, sampling points $Q=\left\{\left.q_{j} \in \Phi\right|_{j=1,2, \ldots}\right\}$ are generated on $\Phi$ with respect to user-defined parameters (Figure 1(c), red points). An average distance $H\left(q_{j}\right)$ of $q_{j} \in Q$ is computed by

$$
H\left(q_{j}\right)=\frac{\sum \lambda_{i} D\left(\forall p_{i} \in U\right)}{\sum \lambda_{i}}
$$

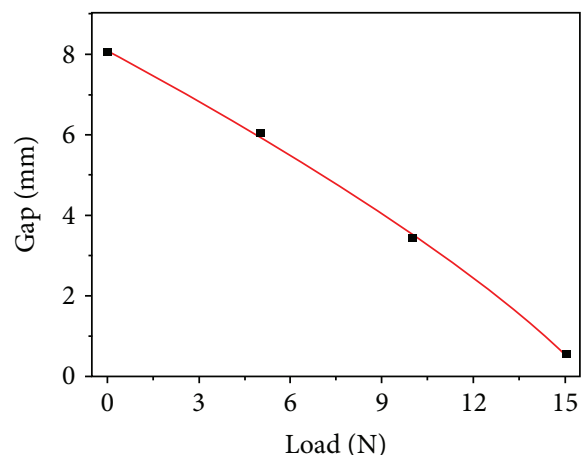

Figure 4: Relationship between load and gap size $\delta$.

TABLe 1: Mechanical properties of T700/YPH-25.

\begin{tabular}{lcc}
\hline Property & Value & Unit \\
\hline$E_{1}$ & 143 & $\mathrm{GPa}$ \\
$E_{2}$ & 8.0 & $\mathrm{GPa}$ \\
$G 12$ & 4.4 & $\mathrm{GPa}$ \\
$G 23$ & 2.8 & $\mathrm{GPa}$ \\
$\mu 12$ & 0.34 & \\
$\mu 23$ & 0.50 & \\
\hline
\end{tabular}

where $U=\left\{p_{m} \in P \mid d\left(f\left(p_{m}\right), q_{j}\right)<r\right\}, d($,$) represents the$ Euclidean distance between two points, $r$ is a user-defined parameter, and $\lambda_{i}$ indicates the coefficient of weight given by

$$
\lambda_{i}=1-\frac{d\left(f\left(p_{i}\right), q_{j}\right)}{2 r} .
$$

The thinking behind (7) is that the less the distance from $f\left(p_{i}\right)$ to $q_{j}$ is, the larger the weight coefficient is. Considering the definition of $U$, it is clear that $\lambda_{i} \in[0.5,1]$.

An example for $H\left(q_{j}\right)$ computation is given in Figure 2 where the red dots represent $\left\{q_{j}\right\}$ and the blue ones indicate $\left\{f\left(p_{i}\right)\right\}$. After a $q_{j}$ is specified (the center), candidates used for computation are determined with respect to the definition of $U$ (dark blue dots inside the red dashed circle). To ensure the accuracy of computation, the number of 


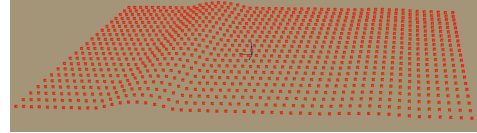

(a)

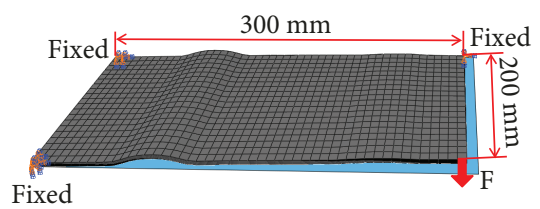

(b)

FIgure 5: Finite element model creation. (a) 2D mesh model. (b) FE model.

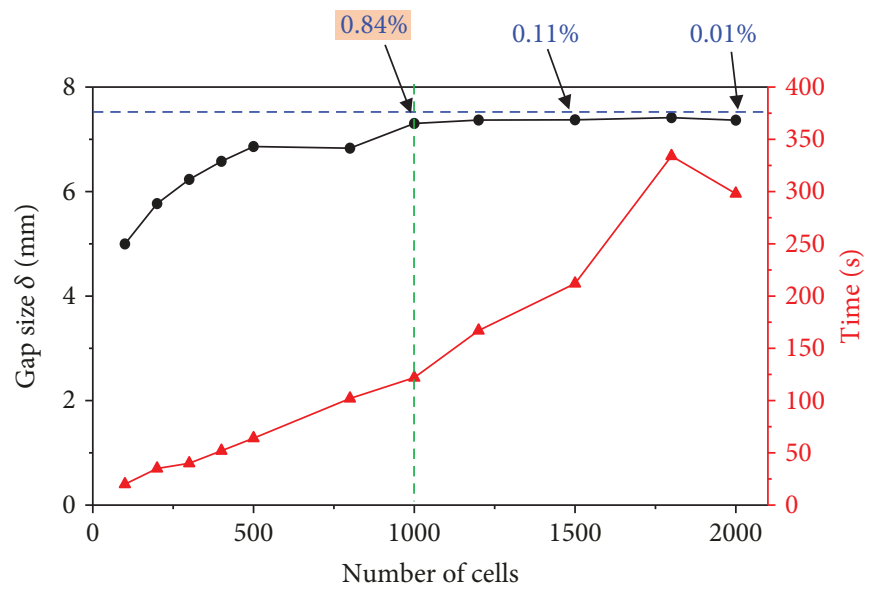

Figure 6: Influence of mesh size on computational accuracy and time.

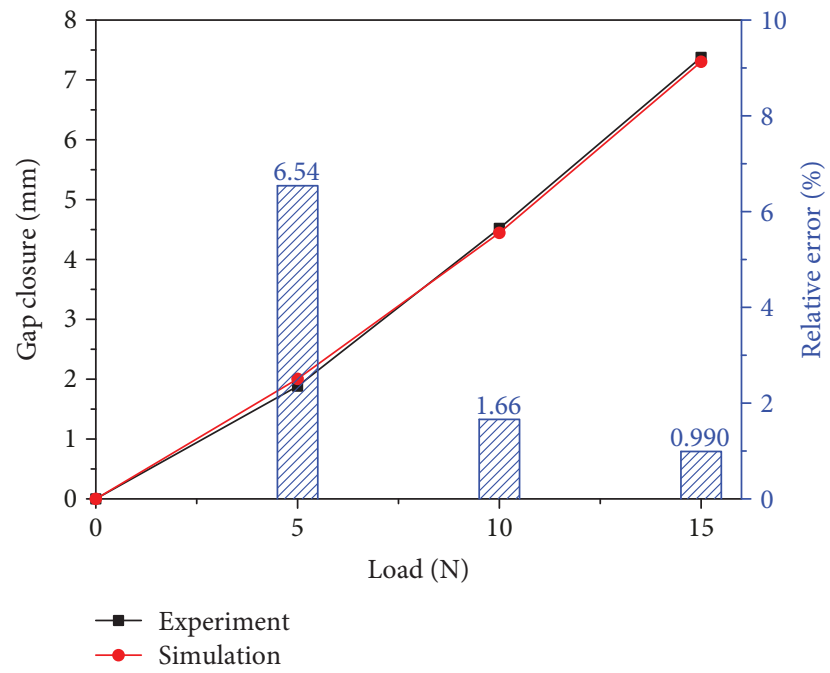

Figure 7: Comparison of simulation and experiment.

candidates \#U is compared to a user-defined variable $\delta$. In case $\# U<\delta$, the parameter $r$ is enlarged by 1.5 times $(r=$ $1.5 r)$ until $\# U \geq \delta$ is met. In this study, $\delta=20$.

All the sampling points $Q$ are then mapped by $g: Q \rightarrow V$, which is given by (8), to generate vertices $V=\left\{\left.g\left(q_{j}\right)\right|_{\forall q_{j} \in Q}\right\}$ used as nodes of finite element meshes.

$$
g: \mathbf{q}_{\mathbf{j}}+\frac{\mathbf{n} \cdot H\left(q_{j}\right)}{\|\mathbf{n}\|} .
$$

$V$ meshes can be generated and used for assembly shape prediction by FEA. Figure 1(d) gives such an example. Note that the meshes were moved far away from the source point cloud so as to show clearly. The meshes can be directly used as an input of a simulation when the plate is modeled by two-dimensional elements in a finite element method. Alternatively, they can be extruded along their normal direction with a specified thickness so as solid elements are created and the plate is then modeled by three-dimensional elements. After the boundary conditions and loads are applied, the shape of the plate in a specified configuration can be predicted.

\section{Method Validation}

For all numerical approaches to assembly gap prediction, the key point is if the compliance and contacts in assemblies are considered. So to validate the proposed method, an experiment is conducted as shown in Figure 3. A rectangular CFRP specimen $(300 \mathrm{~mm} \times 200 \mathrm{~mm})$ with form deviation is fixed on a metallic plate by constraining its three corners with clamps. A loading equipment is used to deform the CFRP specimen, and the load head is set right above the free corner. The distance $\delta$ from the free corner to the metallic plate is measured 3 times for each configuration, and the averaged value is used for simulation result validation. A laser scanner is used to scan the CFRP specimen after the load is applied. Note that considering the size of the specimen deformation caused by gravity is ignored in this study.

Loads of $0 \mathrm{~N}, 5 \mathrm{~N}, 10 \mathrm{~N}$, and $15 \mathrm{~N}$ were applied, and the relationship between the load and $\delta$ is shown in Figure 4. It can be seen that with the increasing of load, the distance $\delta$ 


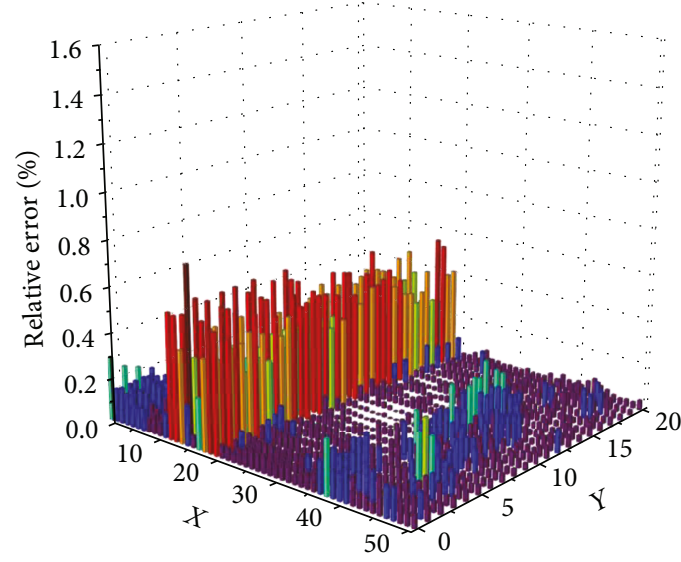

(a)

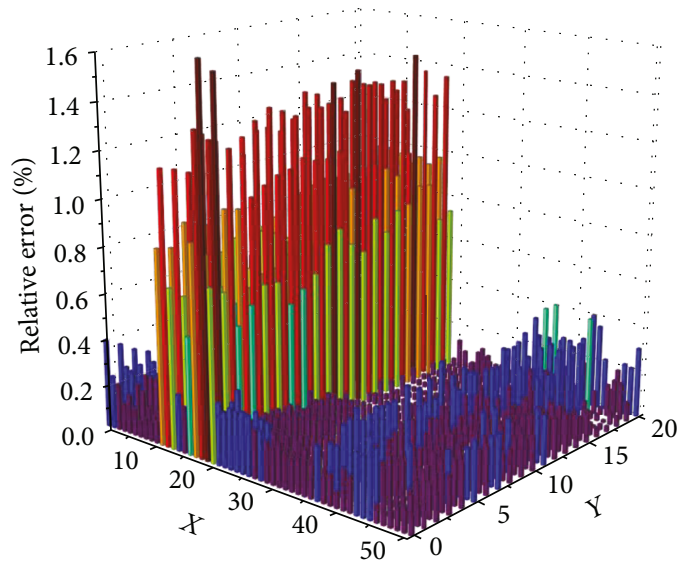

(c)

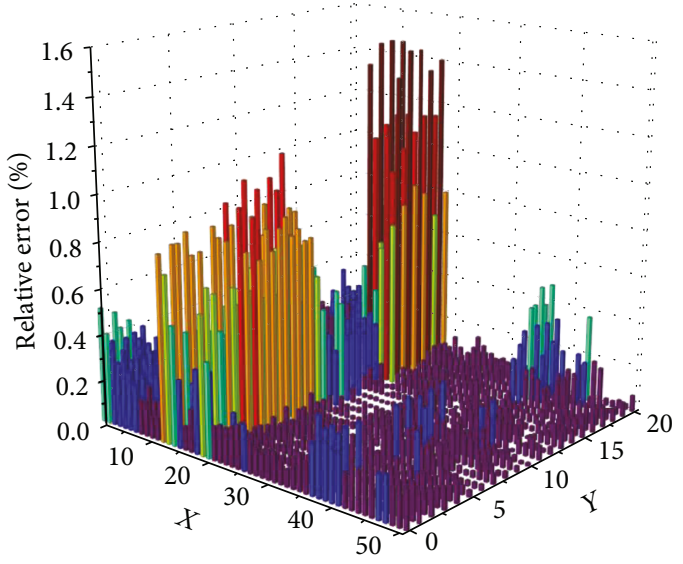

(b)

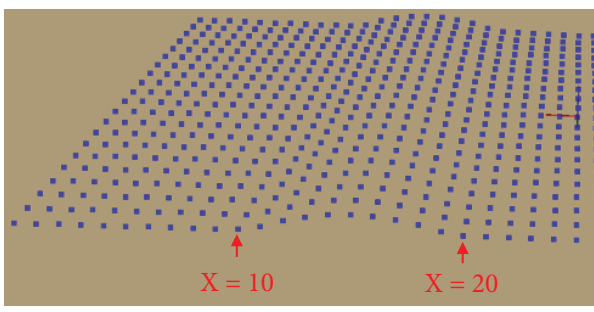

(d)

Figure 8: Comparison of simulation and experiment. (a) 5 N. (b) 10 N. (c) 15 N. (d) Arc area.

decreases and the relationship between them is approximately linear. The specimen was scanned 4 times and used for the proposed method validation.

The CFRP specimen was made by T700/YPH-25 carbon fiber/epoxy prepregs, which has nominal ply thickness of $0.15 \mathrm{~mm}$. Mechanical properties of the material are given in Table 1. The lay-up sequence of the specimen is [45/90/0/90 $/ 0 / 90 / 0 / 90 / 0 / 90 /-45 / 0 / 90 / 0 / 90 /-45 / 90 / 90 / 0]$, and the nominal thickness is $2.85 \mathrm{~mm}$.

Starting from the point cloud taken under $0 \mathrm{~N}$ load and following the procedure given in the previous section, twodimensional meshes are obtained as shown in Figure 5(a). After extruding the mesh $2.85 \mathrm{~mm}$ thickness, solid elements are got and used for assembly shape prediction (Figure 5(b)).

It is well known that the solution precision of FEA is sensitive to mesh size. Generally speaking, the less the mesh size is, the higher the accuracy of the solution will be and the more time the computation will take. In addition, a mesh aspect ratio, i.e., the ratio of the longest to the shortest side in a mesh, should be as possible as small to ensure the best results. So to get mesh-independent simulation result and acceptable solution precision as well, an experiment is done and the influences of mesh size (number of meshes) on computational accuracy and time are shown in Figure 6. Note that the mesh aspect ratio is kept no more than 3 for linear computation. Considering computational accuracy and time, the CFRP specimen is meshed into 1000 meshes.

After applying the boundary conditions and loads, simulations were carried out. The simulation results are compared to experimental ones, and the comparison result is given in Figure 7. It can be seen that when the applied load is $5 \mathrm{~N}$, the relative error between the simulation result and the experimental one is $6.54 \%$. When the load increases up to $15 \mathrm{~N}$, the relative error decreases to $0.99 \%$, which proves the feasibility of the proposed method.

In addition, after the simulations were carried out, the results were output and compared to the experimental ones as shown in Figure 8, where the coordinates $X$ and $Y$ are used to locate the nodes used to create the meshes (red points in Figure 5(a)) and distance ( $Z$-axis) indicates the deviation from the experimental results to the simulation ones at each node.

It can be seen that with the increasing of load, the deviations increase and the largest deviation appeared in the arc area $(10<X<20$, Figure $8(\mathrm{~d}))$. This may be mainly due to the meshing rules used in this study. Generally speaking, intensive meshes should be used for the arc area so as to capture the rapid changing of stress. So adaptive meshes will 
bring us a more accurate result and reduce the deviation as a result. In addition, the reconstruction of experimental result inevitably introduces errors and may consequently enlarge the deviation. Nevertheless, compared to the initial gap size, the deviations are relatively low and the computation results are acceptable for assembly gap assessment.

\section{Conclusion}

A numerical approach to assembly gap prediction of composite structures is presented in this paper. First, assembly components are scanned in a specified configuration and finite element meshes are then generated by using the scanned data. Next, the shape variations of each component are evaluated by finite element analysis after considering the differences between constraints and forces presented in scanning and assembly configurations. Finally, the assembly gaps are assessed by assembling the simulated preassembly shapes of all components. The feasibility of the proposed method is proved by an experiment.

Being a new method, there are still rooms for modification and improvement. First, the meshing rules should be updated so as to minimize the deviation of simulation results for components with irregular shapes. In addition, the mesh generation procedure will be optimized in the future such that the proposed method can be used for large complex structure analysis without taking too much time.

\section{Data Availability}

The point cloud (.obj) data used to support the findings of this study were supplied by Xueshu Liu under license and so cannot be made freely available. Requests for access to these data should be made to Xueshu Liu through liuxs@dlut.edu.cn.

\section{Conflicts of Interest}

The authors declare that there is no conflict of interest regarding the publication of this paper.

\section{Acknowledgments}

This work was supported by the Major State Basic Research Development Program (grant number 2014CB046504), the National Natural Science Foundation of China (grant number 51475073), and the Fundamental Research Funds for the Central Universities (DUT17JC19). The authors would like to acknowledge the above financial supports.

\section{References}

[1] C. Hühne, A.-K. Zerbst, G. Kuhlmann, C. Steenbock, and R. Rolfes, "Progressive damage analysis of composite bolted joints with liquid shim layers using constant and continuous degradation models," Composite Structures, vol. 92, no. 2, pp. 189-200, 2010.

[2] J. X. Dhôte, A. J. Comer, W. F. Stanley, and T. M. Young, "Study of the effect of liquid shim on single-lap joint using 3d digital image correlation," Composite Structures, vol. 96, pp. 216-225, 2013.

[3] J. X. Dhôte, A. J. Comer, W. F. Stanley, and T. M. Young, "Investigation into compressive properties of liquid shim for aerospace bolted joints," Composite Structures, vol. 109, pp. 224-230, 2014

[4] Y. Zhai, D. Li, X. Li, and L. Wang, "An experimental study on the effect of joining interface condition on bearing response of single-lap, countersunk composite-aluminum bolted joints," Composite Structures, vol. 134, pp. 190-198, 2015.

[5] N. M. Chowdhury, J. Wang, W. K. Chiu, and P. Chang, "Experimental and finite element studies of thin bonded and hybrid carbon fibre double lap joints used in aircraft structures," Composites Part B: Engineering, vol. 85, pp. 233-242, 2016.

[6] L. Liu, J. Zhang, K. Chen, H. Wang, and M. Liu, "Experimental and numerical analysis of the mechanical behavior of composite-to-titanium bolted joints with liquid shim," Aerospace Science and Technology, vol. 49, pp. 167-172, 2016.

[7] R. Ascione and W. Polini, "Measurement of nonrigid freeform surfaces by coordinate measuring machine," The International Journal of Advanced Manufacturing Technology, vol. 51, no. 912, pp. 1055-1067, 2010.

[8] G. N. Abenhaim, A. Desrochers, and A. Tahan, "Nonrigid parts' specification and inspection methods: notions, challenges, and recent advancements," The International Journal of Advanced Manufacturing Technology, vol. 63, no. 5-8, pp. 741-752, 2012.

[9] G. N. Abenhaim, A. S. Tahan, A. Desrochers, and R. Maranzana, "A novel approach for the inspection of flexible parts without the use of special fixtures," Journal of Manufacturing Science and Engineering, vol. 133, no. 1, article 011009, 2011.

[10] I. Gentilini and K. Shimada, "Predicting and evaluating the post-assembly shape of thin-walled components via $3 \mathrm{~d}$ laser digitization and fea simulation of the assembly process," Computer-Aided Design, vol. 43, no. 3, pp. 316-328, 2011.

[11] H. Radvar-Esfahlan and S.-A. Tahan, "Nonrigid geometric metrology using generalized numerical inspection fixtures," Precision Engineering, vol. 36, no. 1, pp. 1-9, 2012.

[12] A. Jaramillo, F. Prieto, and P. Boulanger, "Fast dimensional inspection of deformable parts from partial views," Computers in Industry, vol. 64, no. 9, pp. 1076-1081, 2013.

[13] A. Jaramillo, F. Prieto, and P. Boulanger, "Deformable part inspection using a spring-mass system," Computer-Aided Design, vol. 45, no. 8-9, pp. 1128-1137, 2013.

[14] G. N. Abenhaim, A. Desrochers, A. S. Tahan, and J. Bigeon, “A finite-element boundary condition setting method for the virtual mounting of compliant components," Journal of Computing and Information Science in Engineering, vol. 15, no. 4, article 041005, 2015.

[15] G. N. Abenhaim, A. Desrochers, A. S. Tahan, and J. Bigeon, "A virtual fixture using a fe-based transformation model embedded into a constrained optimization for the dimensional inspection of nonrigid parts," Computer-Aided Design, vol. 62, pp. 248-258, 2015.

[16] V. Sabri, S. A. Tahan, X. T. Pham, D. Moreau, and S. Galibois, "Fixtureless profile inspection of non-rigid parts using the numerical inspection fixture with improved definition of displacement boundary conditions," The International Journal 
of Advanced Manufacturing Technology, vol. 82, no. 5-8, pp. 1343-1352, 2016.

[17] S. Sattarpanah Karganroudi, J.-C. Cuillière, V. Francois, and S.-A. Tahan, "Automatic fixtureless inspection of non-rigid parts based on filtering registration points," The International Journal of Advanced Manufacturing Technology, vol. 87, no. 14, pp. 687-712, 2016.

[18] F. Thiebaut, C. Lacroix, L. Andolfatto, and C. Lartigue, "Evaluation of the shape deviation of non rigid parts from optical measurements," International Journal of Advanced Manufacturing Technology, vol. 88, no. 5-8, pp. 1937-1944, 2017.

[19] C. Lacroix, L. Mathieu, F. Thiebaut, M. Douilly, and H. Falgarone, "Numerical process based on measuring data for gap prediction of an assembly," Procedia CIRP, vol. 27, pp. 97-102, 2015. 


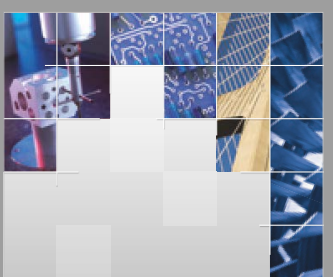

\section{Enfincering}
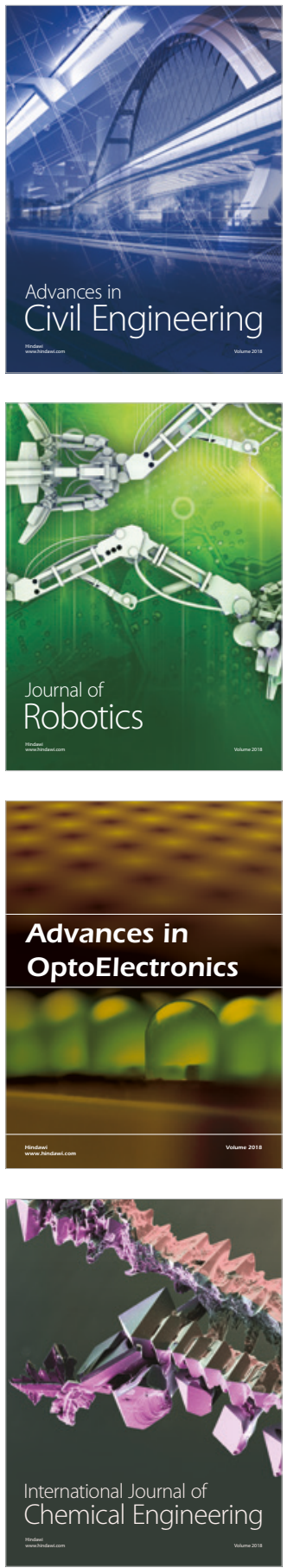

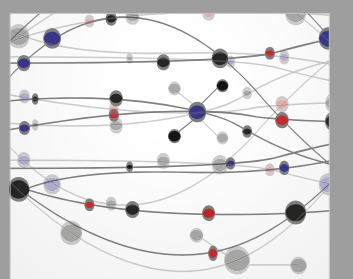

\section{Rotating \\ Machinery}

The Scientific World Journal

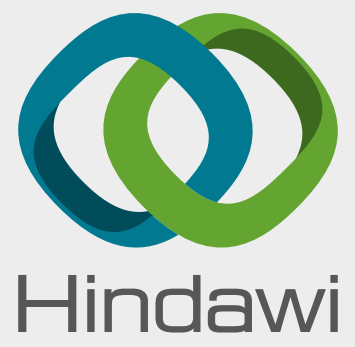

Submit your manuscripts at

www.hindawi.com
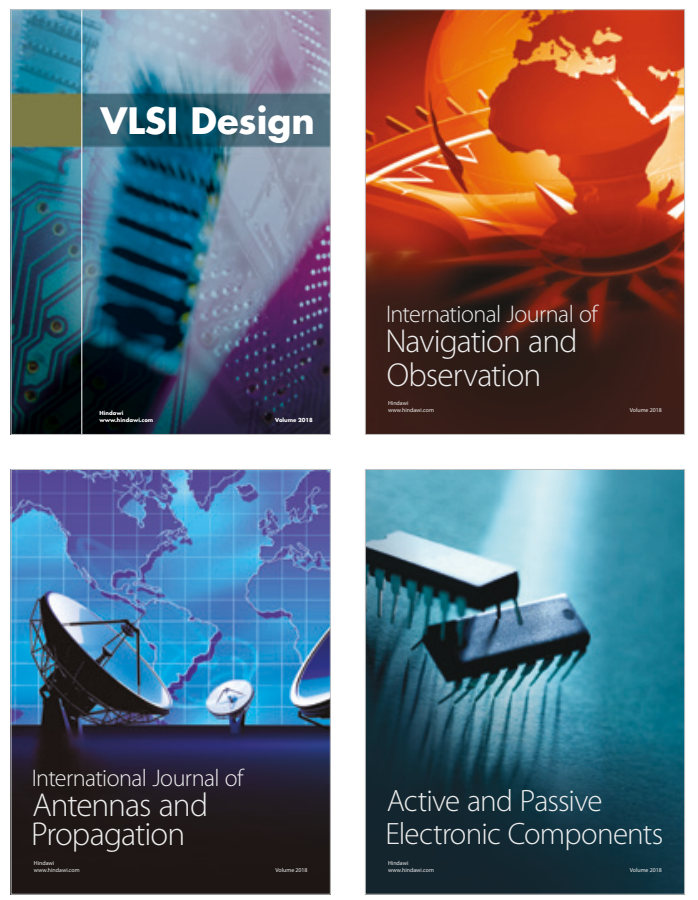
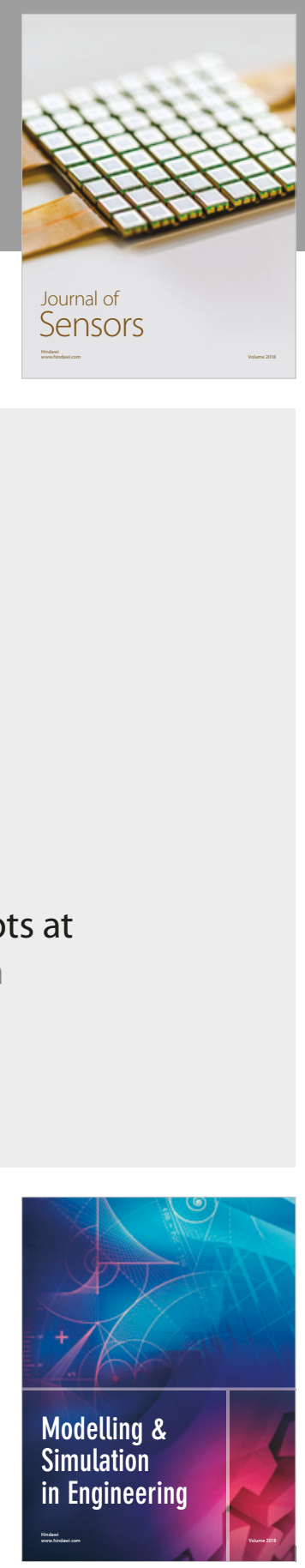

\section{Advances \\ Multimedia}
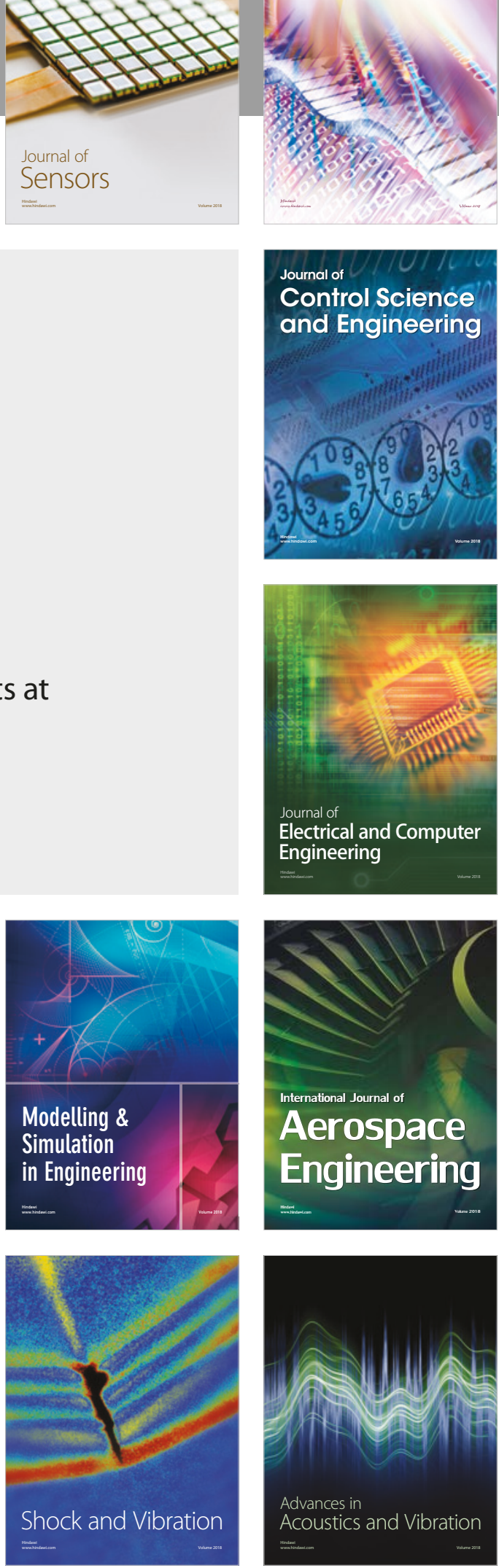